EMBRYARIDDLE
Aeronautical University

SCHOLARLY COMMONS

\section{International Journal of Aviation,} Aeronautics, and Aerospace

\title{
UAS for Public Safety Operations: A Comparison of UAS Point Clouds to Terrestrial LIDAR Point Cloud Data using a FARO Scanner
}

Joseph S. Cerreta

Embry-Riddle Aeronautical University, cerretaj@erau.edu

Scott S. Burgess

WW/Flight, burgesco@erau.edu

Jeremy Coleman

Embry-Riddle Aeronautical University, colemae3@my.erau.edu

Follow this and additional works at: https://commons.erau.edu/ijaaa

Part of the Aviation and Space Education Commons, Criminal Procedure Commons, Law Enforcement and Corrections Commons, Management and Operations Commons, Operational Research Commons, and the Other Aerospace Engineering Commons

\section{Scholarly Commons Citation}

Cerreta, J. S., Burgess, S. S., \& Coleman, J. (2020). UAS for Public Safety Operations: A Comparison of UAS Point Clouds to Terrestrial LIDAR Point Cloud Data using a FARO Scanner. International Journal of Aviation, Aeronautics, and Aerospace, 7(1). https://doi.org/10.15394/ijaaa.2020.1432

This Article is brought to you for free and open access by the Journals at Scholarly Commons. It has been accepted for inclusion in International Journal of Aviation, Aeronautics, and Aerospace by an authorized administrator of Scholarly Commons. For more information, please contact commons@erau.edu. 
Crime scene investigation (CSI) is an involved process and ultimately limits long term access to the scene, which may be vital to commerce. Accordingly, investigators must comprehend the problems it can cause to the impedance of access, and indeed for the families of those involved. Motor vehicle accidents (MVA) are similarly affected and can delay traffic flow for hours as the site is reconstructed, data collected, and then the accident site is cleared (Struble, 2014). This is no different for CSI. The removal of this impedance to regenerate normal flow or daily operation falls upon public safety officials, primarily law enforcement. Reconstruction after data collection of these scene sites has evolved over time, but the goal has always remained the same; a rapid and detailed collection of all data, followed by clearance of the scene to restore normal life (Bullock, Hainje, Habib, Horton, \& Bullock, 2019). Rapid data gathering has long been the challenge to this essential task, and over the years, the tools have evolved.

As Unmanned Aircraft Systems (UAS) proliferate, their utility broadly spans many industries, and crime scene investigation is an area of promising results. The use of three-dimensional point clouds or two-dimensional orthogrammetric data (Ortho) is proving to work incredibly well as evaluative tools that can also stand up in court according to Law Enforcement (LE), from municipal through federal levels according to federal law enforcement agents (personal communication, 26 September 2019). Terrestrial laser scanners are tools for creating baseline models for comparison of datasets to other tools such as satellite imagery, SLR cameras, or UAS (Turner, Lucieer, \& Wallace, 2014). The focus on UAS as a rapid collection platform is of great interest to LE, though as budgets can be a challenge, these agencies can be well informed through the benefit of this research when considering where to start looking for accurate data acquisition aerial platforms.

Accurate data sets that can be quickly acquired and processed for timely assessment aids not only LE reporting and preparation of further legal adjudication but also for families looking for answers. Acquisition of UAS platforms for LE operations are affected by many factors like operational integration considerations and limited resources (Lee, 2016). Departments with limited budget options for UAS selection is a significant challenge where data accuracy is essential and available platform performance is broad.

\section{Purpose}

The purpose of this research was to compare multirotor UAS to determine if there were differences in accuracy and precision compared to a FARO terrestrial laser scanner in a crime scene reconstruction scenario. Also, to compare UAS to provide recommended best practices for selecting aircraft, flying heights, and flight patterns with the highest levels of accuracy. 
UAS registered point clouds generated in Pix4Dmapper Pro from a DJI Mavic Pro, DJI Mavic 2 Enterprise Dual, DJI Inspire 1, DJI Inspire 2, DJI Phantom 4 Professional, Parrot Anafi, and Parrot Bebop 2 flying at 82 feet, 100 feet, 150 feet, 200 feet, and 250 feet respectively in a grid, double grid, circle, and double grid + circle pattern were compared to a FARO laser scanner point cloud using CloudCompare. The UAS point clouds RMS errors were calculated from CloudCompare when registered to the laser scanner pointcloud using the Aeropoint GCP positions as registration points to determine UAS point cloud accuracy. A $\mathrm{M} 3 \mathrm{C} 2$ plugin in CloudCompare was used to calculate the precision errors between points in the UAS point clouds to the FARO point cloud, which was used for UASto-UAS comparisons.

\section{Review of Relevant Literature}

Scene Reconstruction. There are many useful tools for scene reconstruction, and more important is to understand and interpret collected data to an acceptable level (Hosseinyalamdary, 2016). The generally accepted methodology for airborne CSI collection is from a preplanned autonomous flight at an altitude that can collect the most accurate data, returning the best results. Many factors are involved in this process; image overlap, sun angles, obstacle clearance, and flight safety elements all contribute to a viable product that can be used as supporting evidence (Mei, 2019). Post-incident investigation requires the collection of data through accurate perspectives, essential for constructing point clouds or Orthos, and UAS technology continues its evolution in the ability to collect viable digital forensic results (Kovar $\&$ Bollo, 2018). The maneuverability of the UAS above a stationary target is a primary capability of the aerial platform and in congested areas (surface roads and vertical obstacles), this method of collection occurs rapidly as this research supports. As with an MVA, collisions cause much debris and depending on the energy transfer involved, can be linearly displaced, further complicating data collection (Kovar \& Bollo, 2018; Araújo, Mendonça, Fontinele, \& Oliveira, 2019). Analyzing CSI data is much harder and granular as the target data is much smaller.

In most cases, LE officials investigating are generally able to look at a scene and with some or no witnessing feedback, quickly have a general idea of the situation (Katz \& Halámek, 2016). This is possible because it requires skill sets in engineering, art, and of course, experience (Struble, 2014). The addition of an accurate three-dimensional perspective supports the use of these tools for incredible accuracy and confirmation. As enumerated by Lord Kelvin (1883), the knowledge of something came from measurement and expression in numbers.

Clearing a scene quickly is vital for many reasons. The integration of UAS technology has enabled more rapid and accurate data collection for the investigator/reconstructionist (Thivierge, 2012; Katz \& Halámek, 2016; Kovar \& Bollo, 2018). The use of advances in technology and rendering three-dimensional 
modeling can speed the process of accurate measurements of trajectories and marks (impact, gouge, scrub, skid, yaw) (Struble, 2014). The investigation must be unbiased, yet accurate with measurements of marks and deformation, all of which assist in the calculations of impact force, trajectories, and additional environmental evidence (Thivierge, 2012). Angles are important when reconstructing what happened as they help support investigative conclusions (Lyu, Huang, Wu, Duan, \& Li, 2017).

Breakthroughs for UAS CSI work continue. Courts have begun to accept three-dimensional point clouds as evidence as well as the data from the drone itself (Salamh, Karabiyik, \& Rogers, 2019). Using aerial captured data in conjunction with ground-based imagery has also proved successful (Urbanová, Jurda, Vojtíšek, \& Krajsa, 2017). Terrestrial and UAS based three-dimensional point clouds are used to create a digital fly-through video of crime scenes (FARO, n.d.; Pix4D, n.d.). As this technology proliferates, it is logical to assume advancements in digital forensic data collection and processing will follow.

Platform Selection. For many LE entities, budgets are limited, and integration of advanced technology like UAS can be a significant challenge as indicated by a federal law enforcement agent (personal communication, 26 September 2019). Obtaining adequate systems requires practical consideration of what the agency is realistically capable of supporting, including cost, operational conditions, processing times, use restrictions, or point densities (Elsner et al., 2018). Based on the expanded use of UAS in many commercial applications such as building information management which calculates change management over time, or infrastructure inspections to identify impending failures, there is a need to capture data to compile for complete assessment accurately. This enhances the speed of business we see today where technology is integrated (Gabrlik, Cour-Harbo, Kalvodova, Zalud, \& Janata, 2018). Resource decisions are quickly made, or components are acquired to negate possible system failures. Data collection tools must be able to collect accurate data very quickly and the data must be accessible. There are many viable UAS platforms available and for reasonable costs. Many are compatible with standard photogrammetry processing tools such as Pix4D, Precision Mapper, or Metashape.

Point Cloud Accuracy. As the point cloud is a product of photogrammetry whereby remotely collected data (imagery) is compiled into an accurate representation of the scanned object, it is not without error. Accuracy is obtained through a best-obtained alignment of linear, planar, and spherical (or volumetric) structure (Dittrich, Weinman, \& Hinz, 2017). Some of these structures contain noise, or have holes, and thus impart error into the final model. Researchers Fiolka, Rouatbi, and Bender (2017) have explored methodologies to address vertical and horizontal gaps in point clouds. Concurrently, researchers globally (Alidoost \& Arefi, 2017; Dittrich, Weinman, \& Hinz, 2017; Fiolka, Rouatbi, \& Bender, 2017; 
Gabrlik, Cour-Harbo, Kalvodova, Zalud, \& Janata, 2018; Gabara \& Sawicki, 2017; Grenzdörffer, Niemeyer \& Frank, 2015; Slocum \& Parrish, 2017) have been focused on studying, identifying, and overcoming these errors in recent years. The level of accuracy appears not only to be tied to the type of sensor used (LiDAR vs. RGB imagery) but also the ultimate costs of these sensors and UAS platforms collectively.

Several industry/government organizations have been involved in establishing conventional digital forensic investigation guidelines. These are the Association of Chief Police Officers and the National Institute for Science and Technology who established closely-related principles and guidelines for obtaining digital forensic evidence (Roder, Choo, \& Le-Khac, 2018). Based on the available literature, there does not appear to be well-established standards for acquiring forensic information specifically with UAS. Reliance upon collected data for acceptance in court proceedings has been established according to federal law enforcement agents (personal communication, 26 September 2019). Pix4D published five use cases where UAS-acquired data was processed and used by public safety agencies for evidence purposes in court proceedings for public safety and emergency response (Pix4D, 2019).

Many factors relate to building accurate point clouds, not the least of which is collecting data with sufficient overlap. More overlap of imagery leads to more accuracy (Turner, Lucieer \& Wallace, 2014). Process completion times are greatly affected by the number of images included in the dataset (Torres-Sánchez et al., 2018), which was observed in this project. While these factors contribute to accuracy, it must be stated that the type of sensor used is essential. In this research, the higher resolution cameras (20-megapixels) on the DJI Phantom 4 Pro (P4P) and DJI Inspire 2 (I2) had smaller RMS errors compared to the 12-megapixel sensors of the other UAS examined. Other sensors, such as LiDAR, while the most expensive, were the most accurate (Elsner et al., 2018) based upon verified location accuracy.

Three-Dimensional Laser Scanners. This research relied on the accuracy of laser scanning data from the FARO terrestrial scanner. In forensic examinations, terrestrial-based laser devices were found to be ideal systems to capture accurate data for investigations (Liberadski, Adamczyk, Witkowski, \& Sitnik, 2018; Komar, Davy-Low, \& Decker, 2012). They were capable of scanning in all light conditions and some could capture color imagery, were predominately portable, standmounted sensors were resistant to environmental conditions (FARO, 2015; McFadden, 2018). Law enforcement entities using FARO were able to produce compelling presentations in $3 \mathrm{D}$ that have been accepted at trial and led to Grand Jury indictments (Archuleta, n.d.).

Laser scanners were unarguably capable of the most accurate acquisition of forensic data where scanners may be employed as this literature and research 
shows, but are not without limitations. The cost of a FARO scanner system was between \$30-50,000. Logistical support, processing capability, training, and operator qualifications can make a high-end product like a FARO, a resourceintensive acquisition. In establishing operational costs, terrestrial-based systems can challenge a small municipal LE agency. These systems are labor-intensive in setup and movement when acquiring the requisite data.

Contrast this with the use of an airborne platform whereby data collection can be completed in a matter of minutes. From arrival on site, setup, preflight, the aircraft can accomplish a slightly less accurate combination of datasets, then process in a similar time to a terrestrial system. During a recent homicide investigation Florida, a UAS was compared to traditional methods. There were 81\%-time savings (over nine person-hours saved) during the data acquisition and reconstruction process (Galante, 2018). Time savings is a value proposition to public safety organizations. The International Association of Fire Chiefs (2017) recommended analyzing the cost-benefit by public safety agencies to support justification from the savings opportunities during a procurement process. Sufficient aerial systems can be acquired for several thousand dollars and take the space of a small carry on. Of the aircraft examined in this research, cost varied from $\$ 500$ for the Parrot Bebop 2 to $\$ 7,000$ for the DJI Inspire 2 (B\&H Photo, n.d.).

\section{Methods}

The research problem for this study was to determine if UAS point cloud data could be as accurate as terrestrial LiDAR data from a FARO laser scanner. Another goal of the research was to determine which UAS, flying altitude and flight pattern created the most accurate point cloud compared to a terrestrial LiDAR dataset. To understand these problems, the study used two research hypotheses.

\section{Hypotheses}

$\mathrm{H} 1_{0}$ : There are no statistical differences in accuracy between UAS point clouds and a FARO point cloud.

$\mathrm{H}_{1}$ : There are statistical differences in accuracy between UAS point clouds and a FARO point cloud.

$\mathrm{H} 2_{0}$ : There are no statistical differences in point cloud accuracy by the UAS flown. $\mathrm{H} 2_{1}$ : There are statistical differences in point cloud accuracy by the UAS flown.

\section{Study Area}

The study area consisted of a simulated crime scene involving damaged vehicles, located in Prescott, AZ, United States. The area consisted of six vehicles with a varying degree of damage to body part crimpling to and bullet holes in windshields, fenders, doors, and rocker panels to indicating the potential damage a vehicle may obtain during a crime, such as a vehicle chase or involvement in an active shooter incident. Figure 1 depicts the location of the study area. The field elevation was 4410 feet above mean sea level. 


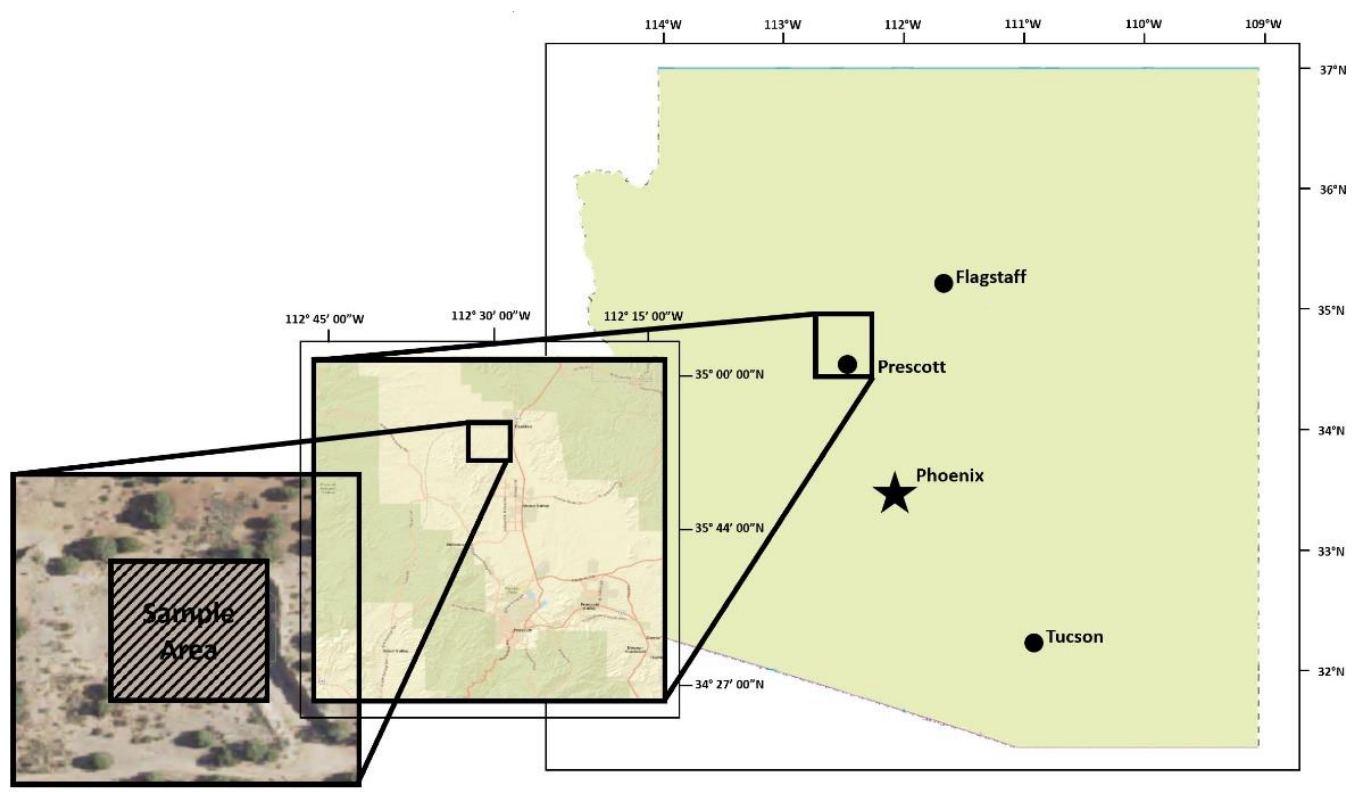

Figure 1. The geographical location of the study location and sample area.

\section{Sample Population}

The area consisted of a $1158 \mathrm{~m}^{2}$ section containing the staged crime scene. A set of 11,235,328 points across the seven UAS using a confidence level of $95 \%$ and a small effect size of 0.10 , yielded a post hoc achieved power of 1.00 . The point cloud root mean square (RMS) errors were recorded from the UAS data compared to the FARO dataset. A combination of t-testing and analysis of variance was used to examine the mean differences between UAS and FARO point cloud points. As depicted in Figure 2, a shapefile was used as a processing area in Pix4Dmapper to confine the extents of the UAS point cloud area to ensure each UAS point cloud was examined over the same area. 


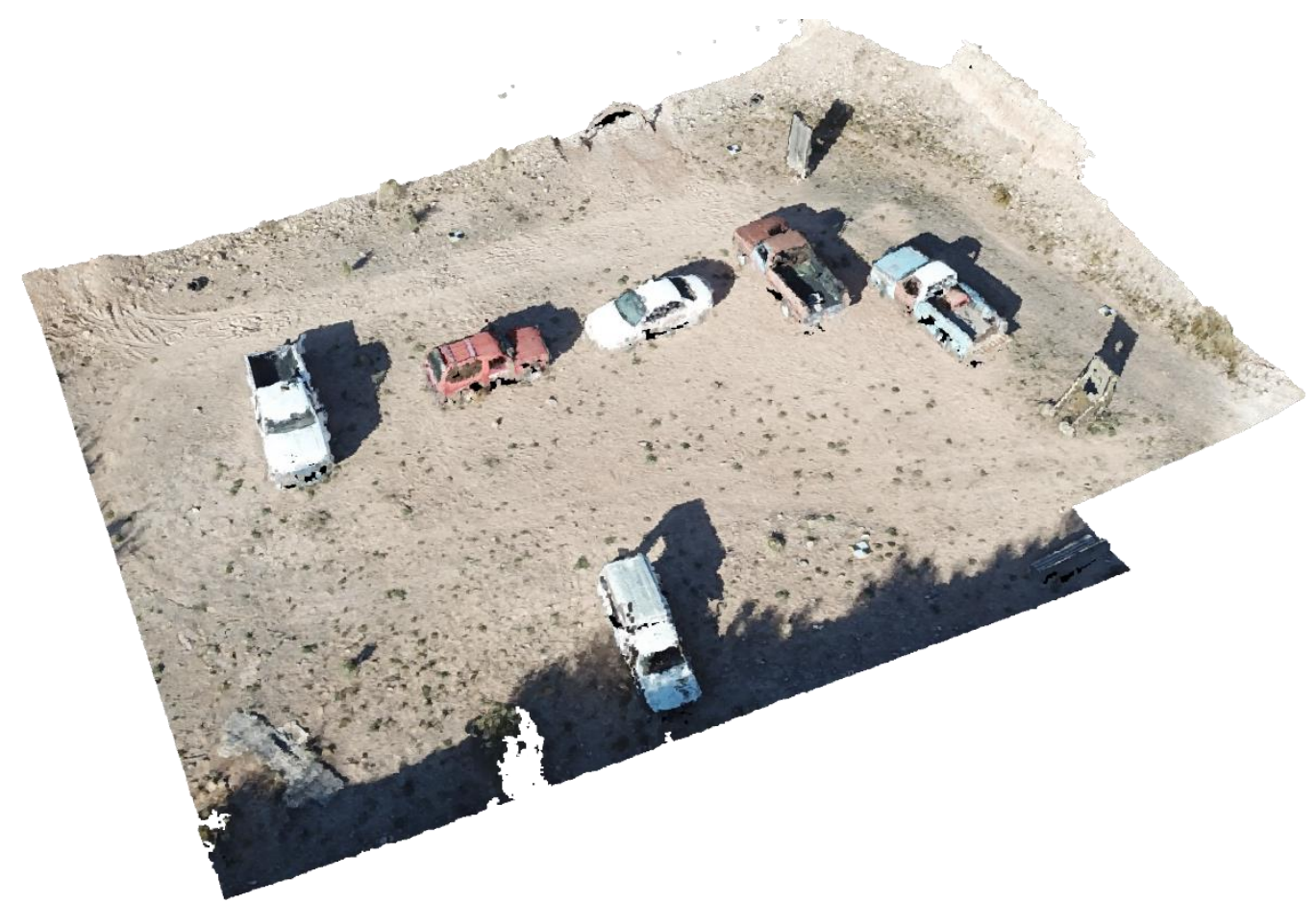

Figure 2. Example point cloud from a DJI Mavic Pro at 200 feet AGL within an $1158 \mathrm{~m}^{2}$ processing area.

\section{Limitations}

This research compared differences between UAS point clouds collected from multiple unmanned aircraft at multiple flying heights in multiple flying patterns and a terrestrial-based FARO laser scanner over a staged crime scene in Arizona. Other UAS or other types of terrestrial LIDAR sensors may have other capabilities or collected in other conditions that could affect similar comparison outcomes. Each UAS was flown sequentially over two days. The FARO scanner data was captured on a separate day. Although there was no movement in the scene between these days, different lighting conditions could have also affected the results. There were varying daylight conditions for each of the flights between one aircraft to the other, causing the sun angle to change between UAS and day of data collection. Ground Control Points (GCPs) were used in the UAS point clouds and a point cloud registration process, using the same GCPs as registration points, was performed in CloudCompare to minimize this variation; however, there may still be effects of the sun angle change not accounted for between flights.

\section{Remotely Sensed Data Collection}

The UAS data collection took place on October 21 and 22, 2019, using a DJI Mavic Enterprise Dual, DJI Mavic Pro, DJI Phantom 4, DJI Inspire 1, DJI Inspire 2, Parrot Anafi, and Parrot Bebop 2. Each UAS flew at 82 feet, 100 feet, 
150 feet, 200 feet, and 250 feet AGL respectively. Additionally, each UAS flew a grid pattern, double grid pattern, and circle pattern at each flying altitude. The number of images varied between UAS, flying altitude, and flight pattern, as shown in Table 1.

Table 1

Quantity of Images for Each UAS at Each Altitude and Flight Pattern

\begin{tabular}{llllllll}
\hline Flight Pattern & M1P & M2ED & I1 & I2 & P4P & Anafi & PB2 \\
\hline 82 Feet Grid & 45 & 102 & 35 & 45 & 39 & 32 & 23 \\
82 Feet Double Grid & 85 & 338 & 55 & 119 & 88 & 100 & 43 \\
82 Feet Circle & 36 & 50 & 71 & 36 & 35 & 45 & 10 \\
82 Feet Double \& Circle & 121 & 219 & 91 & 155 & 123 & 145 & 53 \\
100 Feet Grid & 32 & 68 & 24 & 40 & 28 & 27 & 19 \\
100 Feet Double Grid & 76 & 82 & 47 & 62 & 66 & 84 & 22 \\
100 Feet Circle & 34 & 51 & 36 & 36 & 35 & 38 & 10 \\
100 Feet Double \& Circle & 110 & 66 & 83 & 98 & 101 & 122 & 32 \\
150 Feet Grid & 18 & 44 & 15 & 24 & 15 & 15 & 16 \\
150 Feet Double Grid & 47 & 64 & 26 & 47 & 37 & 40 & 29 \\
150 Feet Circle & 36 & 46 & 36 & 36 & 35 & 26 & 11 \\
150 Feet Double \& Circle & 83 & 55 & 62 & 83 & 72 & 66 & 40 \\
200 Feet Grid & 15 & 14 & 12 & 14 & 12 & 11 & 19 \\
200 Feet Double Grid & 29 & 50 & 23 & 30 & 26 & 30 & 31 \\
200 Feet Circle & 35 & 62 & 35 & 35 & 36 & 18 & 16 \\
200 Feet Double \& Circle & 64 & 56 & 58 & 65 & 62 & 48 & 47 \\
250 Feet Grid & 12 & 12 & 9 & 15 & 12 & 7 & 8 \\
250 Feet Double Grid & 23 & 16 & 11 & 30 & 49 & 23 & 8 \\
250 Feet Circle & 35 & 72 & 35 & 24 & 59 & 19 & 15 \\
250 Feet Double \& Circle & 58 & 44 & 46 & 54 & 54 & 42 & 27 \\
\hline Note. Val
\end{tabular}

Note. Values shown are the number of images. M1P is the DJI Mavic Pro, M2ED is the DJI Mavic 2 Enterprise Dual; I1 is the DJI Inspire 1; I2 is the DJI Inspire 2 equipped with an X5S and 15mm 1.7 ASPH lens; P4P is the DJI Phantom 4 Professional; Anafi is the Parrot Anafi; and BP2 is the Parrot Bebop 2.

Five Aeropoints (Aeropoints, n.d.) were used as GCPs and were emplaced throughout the scene. All the data from all five points were uploaded to the Aeropoints server. Fully-Automated processing in correction-network-coveragearea method of processing was used. The Aeropoints GNSS system connected to a virtual reference network at 40km away and had a mean-variance of $20 \mathrm{~mm}$. All five GCPs were imported to Pix4Dmapper Pro and used as 3D GCPs. 
The Pix4Dcapture mobile application software (Pix4D, version 4.3.31) was used to plan the UAS flights, shown in Figure 3, for all UAS except the DJI M2ED. The M2ED control station was a DJI Smart Controller, on which Pix4Dcapture would not run. For the M2ED, the embedded DJI mission flight planner as a part of the DJI Pilot app was used with a custom camera setting using the camera specifications of sensor size, sensor dimensions, and focal length from DJI (DJI, n.d.). The flight altitudes were set at 82 feet, 100 feet, 150 feet, 200 feet, and 250 feet, respectively. The same 150-foot x 150-foot area was used for each grid and double grid pattern, while the circle flying pattern had a 190-foot x 184-foot area. Flight planning parameters were set with an $80 \%$ longitudinal and $70 \%$ lateral overlap ratio for the grid and double grid pattern. For the grid patterns, the camera depression angle was set at -90 degrees (nadir). For the double grid patterns, the angle was set to -70 degrees (oblique). An image capture angle of $10^{\circ}$ was used for the circle patterns with the camera pointing to the center of the scene. The camera was set to trigger automatically.

The DJI Mission Flight application for the M2ED was set to $80 \%$ longitudinal and $70 \%$ lateral overlap with a $25 \mathrm{~m}$ margin. The camera depression angle was set to -90 degrees for the grid pattern, -60 degrees for the double grid pattern and the camera was pointed at the center of the scene for the circle patterns. The camera was automatically triggered. 


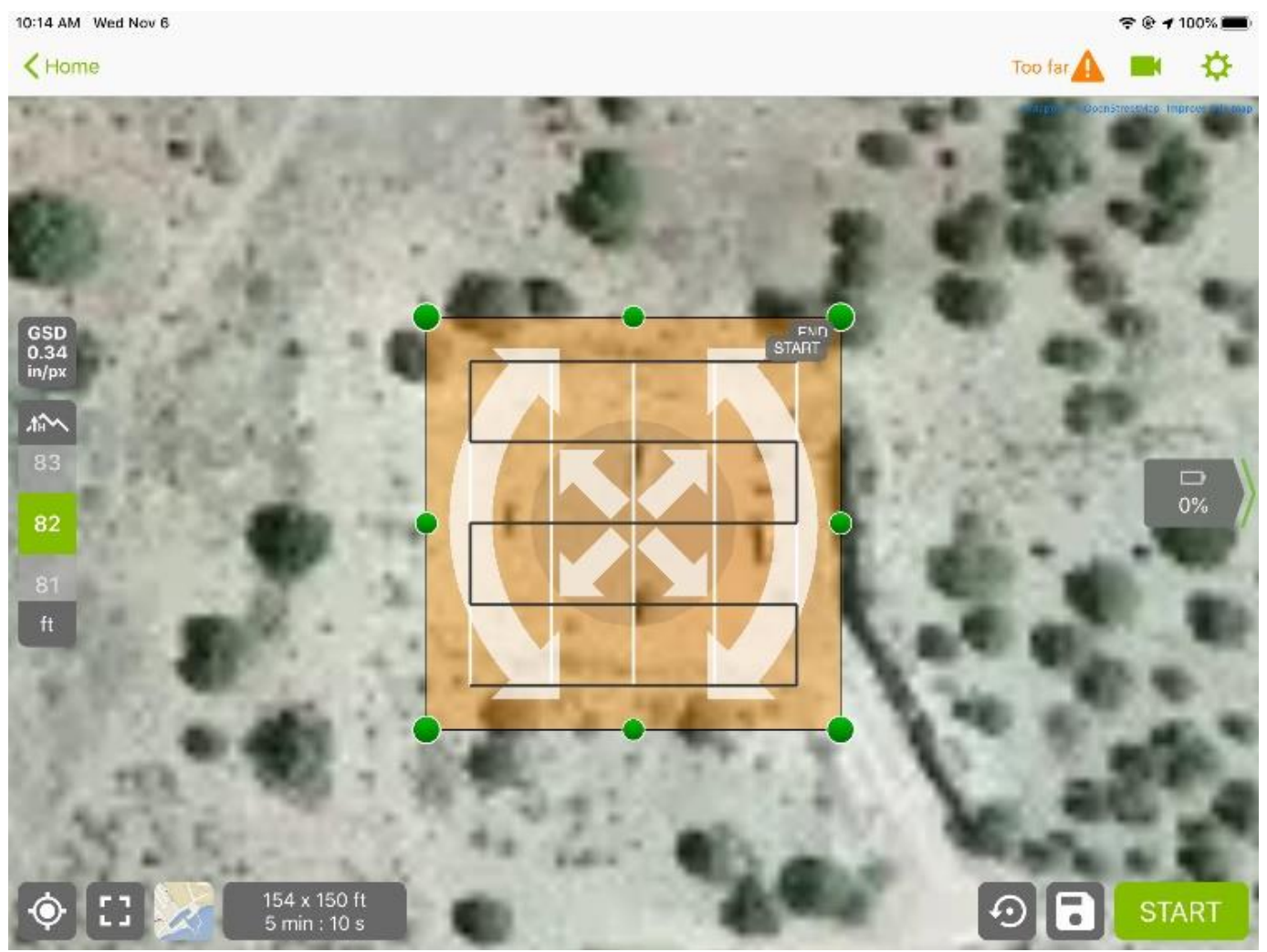

Figure 3. Pix4Dcapture mission plan for the DJI Mavic Pro with 82 feet double grid pattern.

\section{FARO}

The ground data was collected on November 4th, 2019 using a FARO Focus S70 Laser Scanner. There were 20 scans taken around the scene from varying heights. Placement around the scene is shown in Figures 4 and 5. The system was set on $1 / 4$ resolution with three times quality giving a point distance of $6.1 \mathrm{~mm}$ apart at $10 \mathrm{~m}$. At three times quality, the scan repeats three times to verify the location of each point giving greater accuracy to the rendered scans. Each scan took 66 pictures at the end to help with the color balance of the rendered scene. The images were also used to create the planar view which aided with registering the scans. The planar view was used to take measurements of the scene; however, the measurement data came from the underlying point cloud. In order to recreate the UAS images, the same five GCPs were used in the scene. 


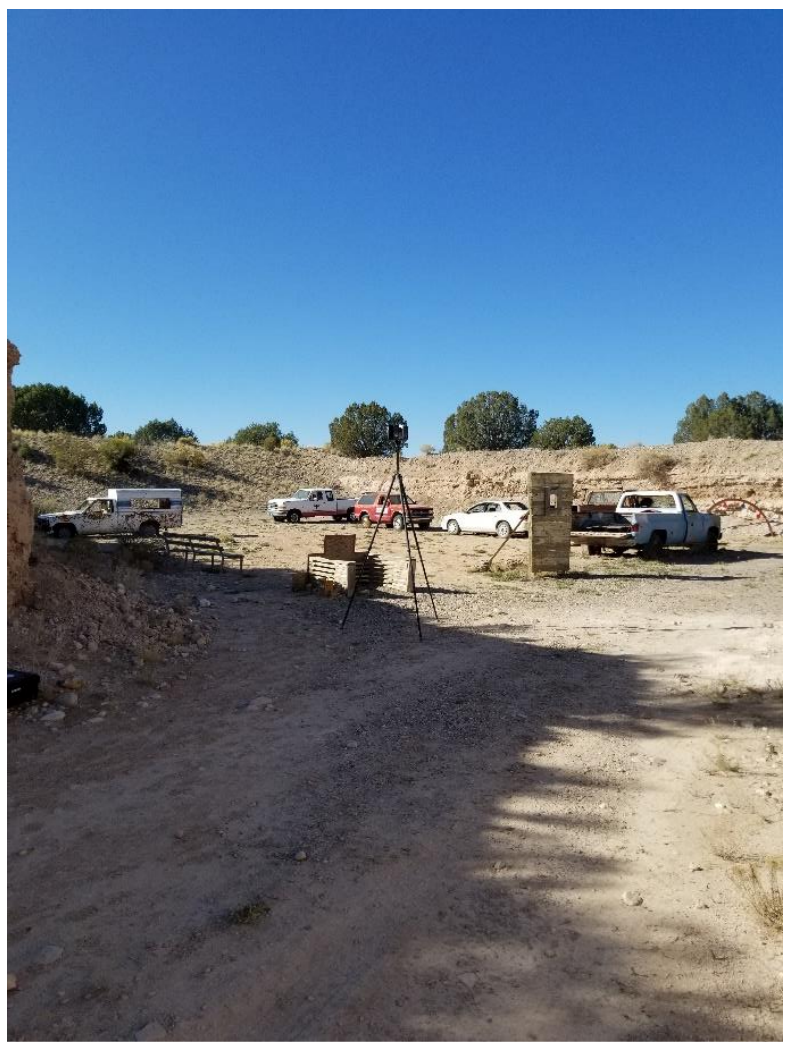

Figure 4. FARO scanner emplaced at the staged crime scene. Prescott, AZ. November 4, 2019.

The scans were processed using FARO SCENE software (FARO, n.d.). The software took all the scans and images taken from the scene and built a threedimensional point cloud of the area. The software examined items in multiple scans using the laser data and the images it took to register or overlap the scans. SCENE also examined for targets throughout the scene to increase the accuracy of the registration. Targets were items added to the scene such as specific size reflective spheres or checkerboards. The GCPs were also used to increase the accuracy of the registration process. Manual verification of the registration was performed after the point cloud for the entire scene was generated. The laser scanner collection resulted in a point cloud of $184,381,887$ points with a registration accuracy of $2.6 \mathrm{~mm}$. Registration accuracy was the same as the accuracy calculated by the SCENE software. 


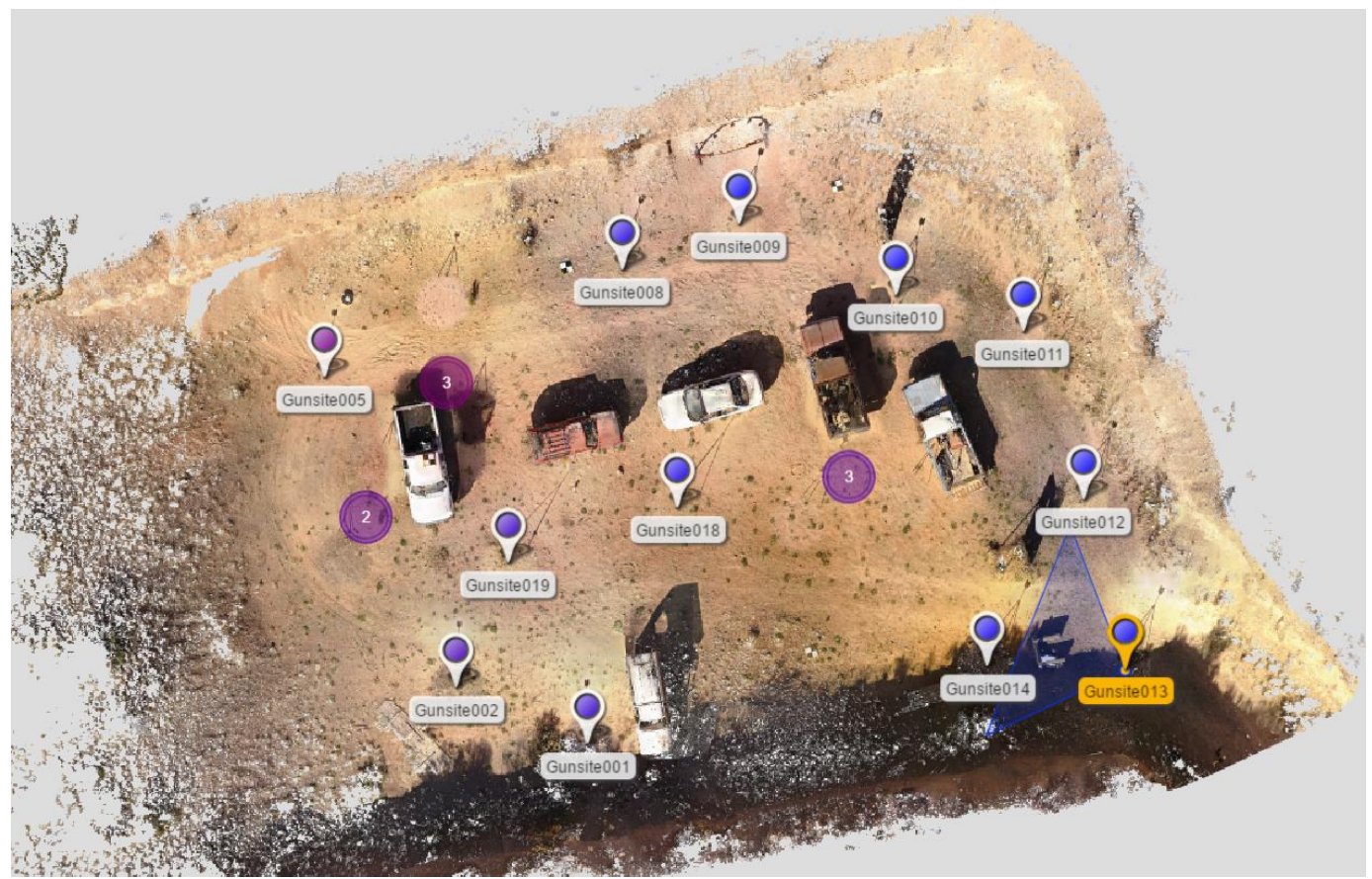

Figure 5. FARO Scanner placement in the sample area.

\section{Image Processing}

Each set of UAS images was processed in Pix4Dmapper Pro separately. Table 2 reflects the Pix4Dmapper Pro processing options for all UAS point cloud datasets. A shapefile of the sample area boundaries was selected as a processing area to keep the UAS point clouds at the same dimension, regardless of UAS, flying altitude, or flight pattern. Using the same shapefile between all datasets enabled the exact geolocation extents of a processing area for all UAS datasets. 
Table 2

Pix4Dmapper Pro Processing Options

\begin{tabular}{ll}
\hline \multicolumn{1}{c}{ Processing Option } & \multicolumn{1}{c}{ Setting } \\
\hline Keypoints Image Scale & Full \\
Image Matching Pairs & Aerial Grid or Corridor \\
Targeted Number of Keypoints & Automatic \\
Calibration Method & Standard \\
Pointcloud Image Scale & Original Image Size \\
Pointcloud Density & High \\
Pointcloud Minimum Matches & 3 \\
Generate Textured Mesh & No \\
Pointcloud Export & XYZ, Merge Tiles Into One File \\
\hline
\end{tabular}

Note. Processing options in Pix4D originated from the 3D Maps template, then tailored to only generate and export a pointcloud.

\section{Point Cloud Registration and Comparison}

After processing in Pix4D, the UAS point clouds were imported into the CloudCompare software. The FARO point cloud was also imported into CloudCompare. Each UAS point cloud was finely registered to the FARO point cloud, using the UAS point cloud as the alignment dataset and the FARO point cloud at the reference dataset. An RMS difference of 1.0e-5, 10\% final overlap, 50,000-point random sampling limit, rotation XYZ, and translation across $T_{x}, T_{y}$, and $\mathrm{T}_{\mathrm{z}}$ were used as registration parameters. The final RMS error of each UAS point cloud registration was recorded.

The points from each UAS point cloud were compared to the FARO, using the M3C2 plugin in CloudCompare (Lague, Brodu, \& Leroux, 2013). The plugin was an algorithm using a method called Multiscale Model to Model Cloud Comparison (M3C2) (Lague, et al., 2013). The algorithm calculated distances of points between point clouds, taking into consideration three-dimensional variation in surface orientation and estimates (Lague, Brodu, \& Leroux, 2013). According to James, Robson, and Smith (2017), the M3C2 algorithm is uniquely suited for calculating point cloud distances of point clouds generated by structure-frommotion (SfM) photogrammetry software. Pix4Dmapper, which is an SfM-based photogrammetry software, was used in this research to generate the UAS point cloud data.

To calculate the distances between points in the point clouds, each UAS point cloud was designated as cloud \#1 and the FARO point cloud with an accuracy of 2.6mm (0.0085 feet) was designated as cloud \#2. Cloud \# 1 was subsampled at a rate of 0.811700 , yielding a mean subsample of 19,509 core points in the UAS point clouds. Subsampling sped up the distance calculations, without significantly 
affecting the measurement accuracy (CloudCompare, n.d.). The RMS error from the previous step was used in the $\mathrm{M} 3 \mathrm{C} 2$ distance calculations for a confidence computation of each point. CloudCompare generated a subsampled file with the calculated distances from each UAS point cloud dataset to the FARO point cloud dataset. From these outputs, statistical analysis was performed to determine statistical differences between the UAS and FARO point cloud data as well we between UAS to develop a recommended best practice aircraft, flying altitude, and flight patterns with the most accurate results.

\section{Results}

UAS registered point clouds generated in Pix4Dmapper Pro from a DJI Mavic Pro, DJI Mavic 2 Enterprise Dual, DJI Inspire 1, DJI Inspire 2, DJI Phantom 4 Professional, Parrot Anafi, and Parrot Bebop 2 flying at 82 feet, 100 feet, 150 feet, 200 feet, and 250 feet respectively in a grid, double grid, circle, and double grid + circle pattern were compared to a FARO laser scanner point cloud using CloudCompare. The UAS point clouds RMS errors were calculated within CloudCompare when registered to the laser scanner point cloud using the Aeropoint GCP positions as registration points to determine UAS point cloud accuracy. An $\mathrm{M} 3 \mathrm{C} 2$ plugin in CloudCompare was used to calculate the precision errors between points of the UAS point clouds to the FARO point cloud, which was used for UASto-UAS comparisons.

\section{UAS Point Cloud Differences to FARO Point Cloud}

A one-sample t-test was conducted to determine if statistically significant differences existed in RMS errors between UAS point clouds and the FARO point cloud. The UAS point cloud accuracy $(M=33.2 \mathrm{~mm}, S D=6.4 \mathrm{~mm})$, compared to the FARO point cloud $t(139)=56.5, p=0.00$. As depicted in Table 3 , The test revealed that there was a significant difference in UAS point cloud accuracy compared to the FARO point cloud accuracy of $2.6 \mathrm{~mm}$. These results suggest there was enough evidence to reject the null hypothesis; there was a significant difference between each UAS point cloud and the FARO point cloud.

Table 3

One-Sample T-Test and CI: UAS Point Cloud RMSE when Registered to FARO Point Cloud

\begin{tabular}{cccc}
\hline $\mathrm{N}$ & $M$ & $S D$ & $S E$ Mean \\
\hline 140 & 33.21 & 6.41 & 0.54 \\
Difference & \multicolumn{2}{c}{$95 \%$ CI for Difference } & \\
30.61 & \multicolumn{2}{c}{$(29.54,31.68)$} & \\
\hline T-Value & DF & $p$-Value & \\
56.54 & 139 & 0.000 &
\end{tabular}

Note: Units are in millimeters. FARO point cloud accuracy was $2.6 \mathrm{~mm}$. 


\section{UAS Point Cloud Results by Flying Height}

A one-way ANOVA was conducted to compare the effect of the RMS error of UAS point clouds by flying height when registered to the FARO point cloud in CloudCompare. An analysis of variance showed an effect of UAS point clouds on FARO point cloud was significant, $F(4,135)=6.66, p=0.000$, see Figure 6 . As indicated in Table 4 and Figure 7, a post hoc Tukey test showed that two flying height groups (Group A: 82 feet, 100 feet, and 150 feet; Group B: 200 feet and 250 feet) differed significantly at $\mathrm{p}<.05$; however, there were was no significant difference by flying height within-group A. While not significant within-group A, the RMS error was smaller as the flying height decreased.

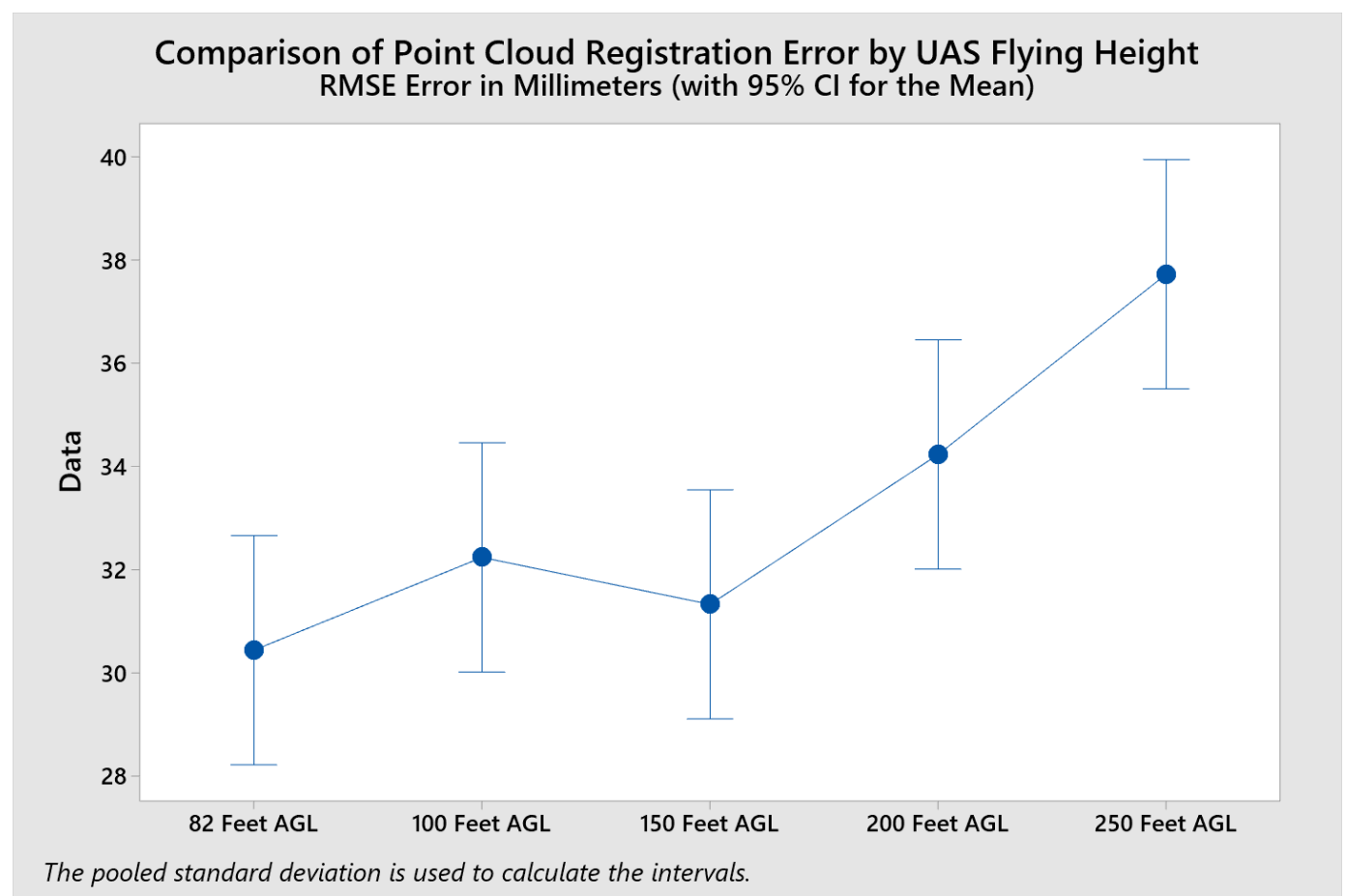

Figure 6. RMS error (in millimeters along the Y axis) of UAS point clouds by flying height when registered to the FARO point cloud. 
Table 4

\section{Grouping Information Using Tukey Method and 95\% Confidence}

\begin{tabular}{cccll}
\hline Flying Height & N & Mean $(\mathrm{mm})$ & Grouping \\
\hline 82 Feet AGL & 28 & 30.44 & A & \\
100 Feet AGL & 28 & 31.24 & A & \\
150 Feet AGL & 28 & 32.24 & A & \\
200 Feet AGL & 28 & 34.23 & A & B \\
250 Feet AGL & 28 & 37.72 & & B \\
\hline
\end{tabular}

Note: Groups A and B are significantly different.

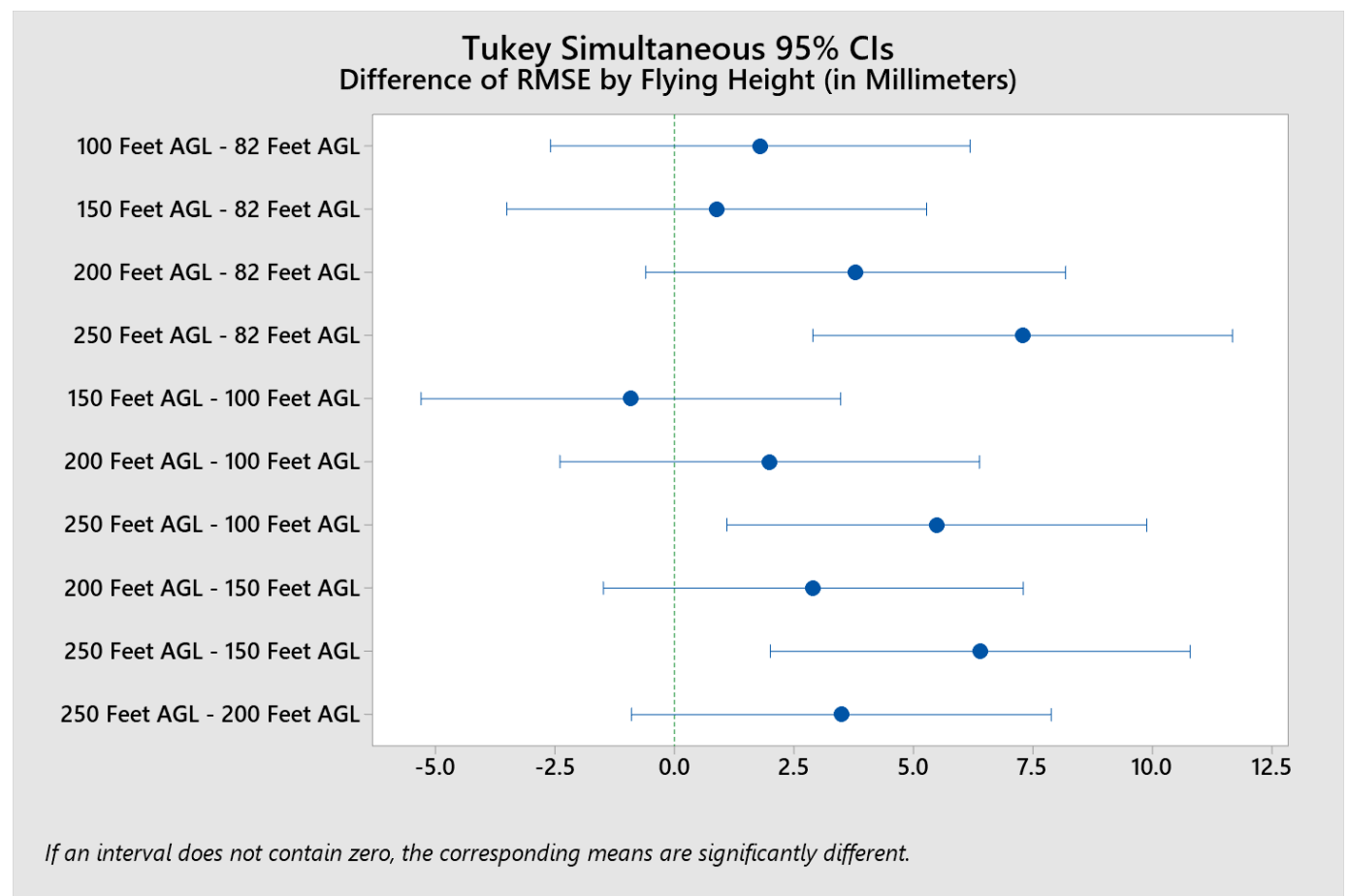

Figure 7. Tukey test results showing the differences in RMS error between UAS flying heights. Differences are in millimeters.

To determine which flying height has the greatest precision to the FARO point cloud, a point distance information between UAS point clouds and the FARO point cloud was performed using the $\mathrm{M} 3 \mathrm{C} 2$ plugin in CloudCompare. A comparison of point distances between the UAS point clouds and the FARO point cloud enabled a determination of which flying height most closely compared (greatest precision) to the FARO dataset. The subsampled core points from each UAS point cloud was grouped by flying height. The $\mathrm{M} 3 \mathrm{C} 2$ plugin calculated the 
distance from each point in the UAS point to the closest point in the FARO point cloud and assigned a distance value.

A one-way ANOVA was conducted to compare the effect of the calculated point distances of UAS points by flying height to the FARO points using the M3C2 plugin in CloudCompare. An analysis of variance showed an effect of UAS point clouds on FARO point cloud was significant, $F(4,2739223)=551.62, p=0.000$, see Figure 8. The test results indicate there was a significant difference in the calculated distance by flying height. The 100 feet flying height had the smallest mean difference in calculated points between the UAS-generated point clouds and the FARO point cloud.

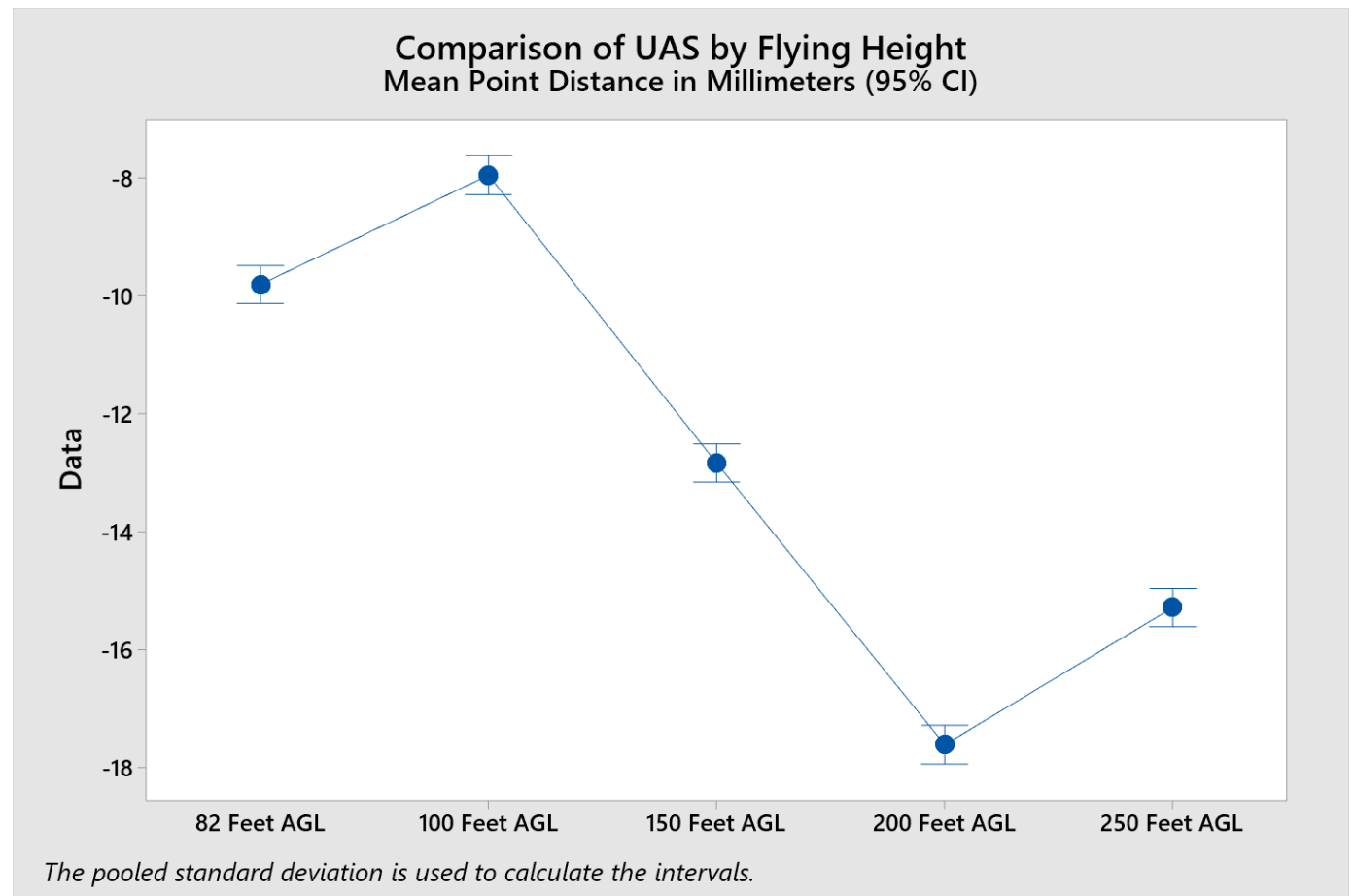

Figure 8. ANOVA test results showing the mean point distance calculation between UAS flying heights to the FARO points. Differences are in millimeters. Points higher in the chart reflect a smaller calculated mean distance by flying height.

\section{UAS Point Cloud Results by Flight Pattern}

A one-way ANOVA was conducted to compare the effect of the RMS error of UAS point clouds by flight pattern when registered to the FARO point cloud in CloudCompare. An analysis of variance showed an effect of UAS point clouds on FARO point cloud was significant, $F(3,136)=4.21, p=0.007$, see Table 5. As shown in Figure 9, the point clouds with the double grid + circle flight pattern had the smallest RMS error when registered to the FARO point cloud. 
Table 5

Descriptive Statistics of UAS Point Cloud Accuracy (RMS Error) by Flight Pattern

\begin{tabular}{lcccl}
\hline Flight Pattern & N & $M$ & $S D$ & \multicolumn{1}{c}{$95 \%$ CI } \\
\hline Double Grid + Circle & 35 & 31.15 & 4.35 & $(29.076,33.215)$ \\
Circle & 35 & 31.59 & 3.84 & $(29.524,33.663)$ \\
Double Grid & 35 & 34.52 & 8.85 & $(32.45,36.59)$ \\
Grid & 35 & 35.50 & 6.43 & $(33.43,37.57)$ \\
\hline
\end{tabular}

Note. Measurements are in millimeters.

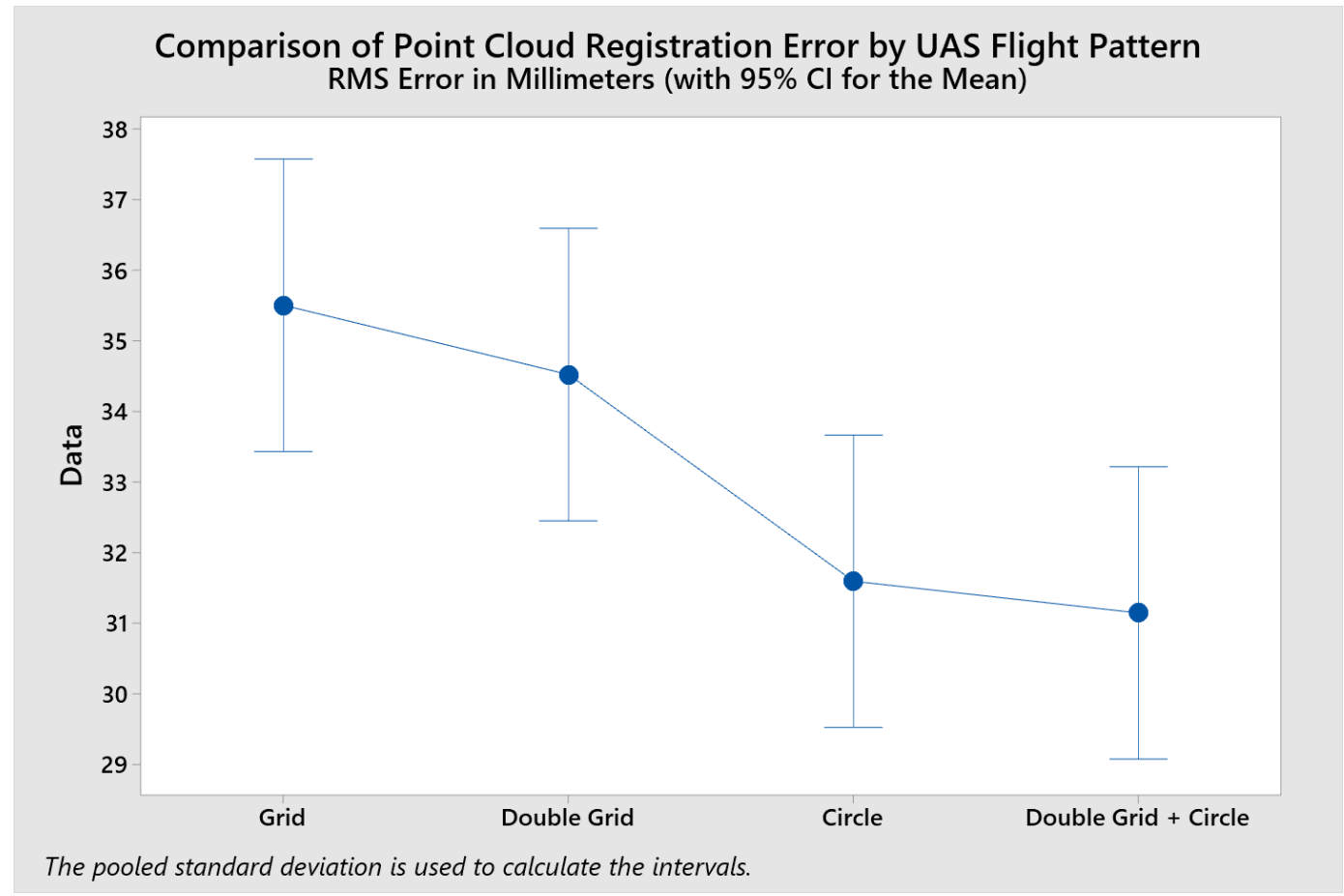

Figure 9. RMS error (in millimeters along the $\mathrm{Y}$ axis) of UAS point clouds by flight pattern when registered to the FARO point cloud.

To determine which flight pattern had the greatest precision to the FARO point cloud, a point distance information between UAS point clouds and the FARO point cloud was performed using the $\mathrm{M} 3 \mathrm{C} 2$ plugin in CloudCompare. A comparison of point distances between the UAS point clouds and the FARO point cloud enabled a determination of which flight pattern most closely compared (greatest precision) to the FARO dataset. The subsampled core points from each UAS point cloud was grouped by flight pattern. The $\mathrm{M} 3 \mathrm{C} 2$ plugin calculated the 
distance from each point in the UAS point to the closest point in the FARO point cloud and assigned a distance value.

A one-way ANOVA was conducted to compare the effect of the calculated point distances of UAS points by flight pattern to the FARO points using the M3C2 plugin in CloudCompare. An analysis of variance showed an effect of UAS point clouds on FARO point cloud was significant, $F(3,2739224)=1183.07, p=0.000$, see Figure 10. The test results indicate there was a significant difference in the calculated distance by flight pattern. The double grid + circle flight pattern had the smallest mean difference in calculated points between the UAS-generated point clouds and the FARO point cloud.

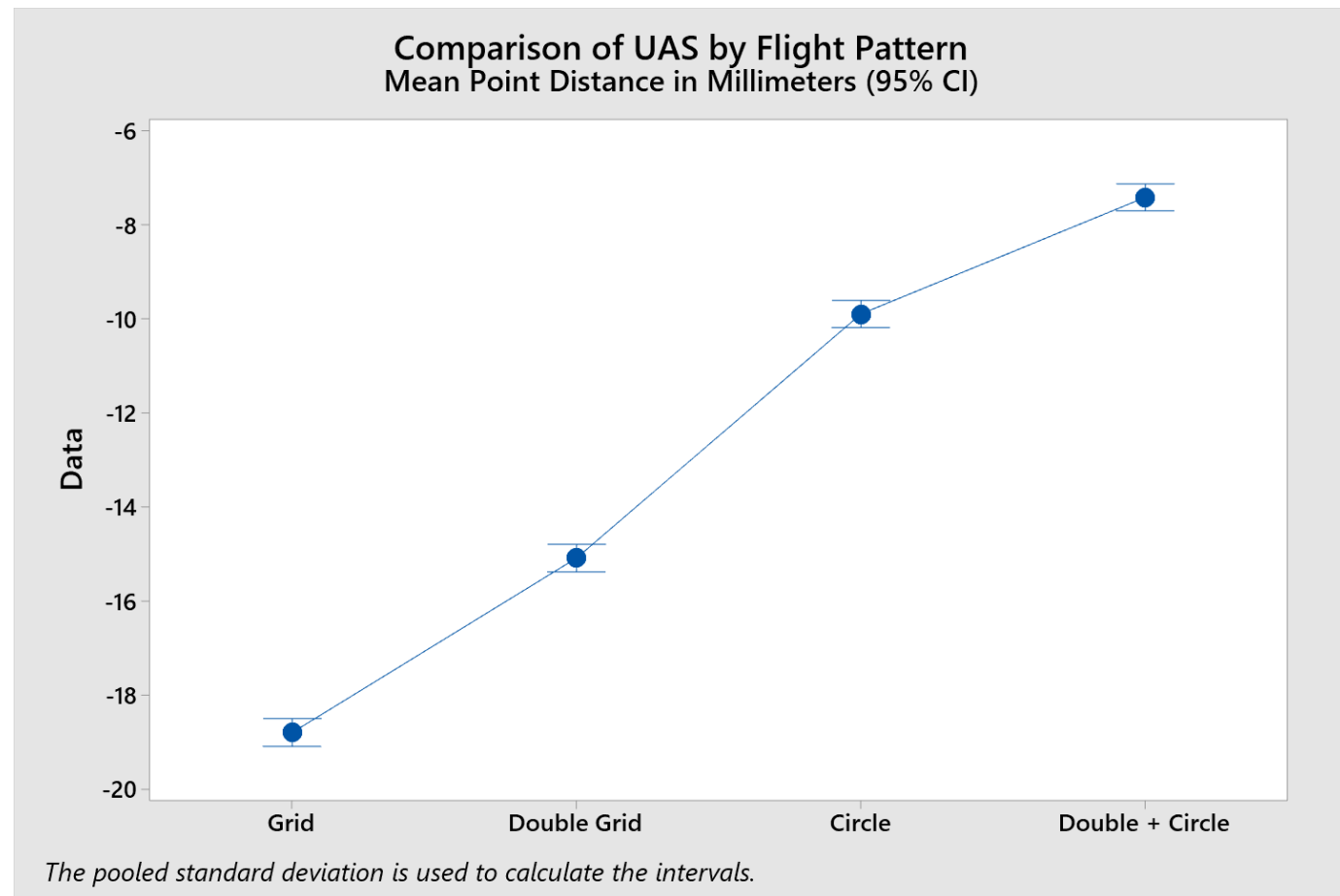

Figure 10. ANOVA test results showing the mean point distance calculation between UAS flight pattern to the FARO points. Differences are in millimeters. Points higher in the chart reflect a smaller calculated mean distance by flight pattern.

\section{UAS Point Cloud Results by Aircraft}

A one-way ANOVA was conducted to compare the effect of the RMS error of UAS point clouds by aircraft model when registered to the FARO point cloud in CloudCompare. An analysis of variance showed an effect of UAS point clouds on FARO point cloud was significant, $F(6,133)=4.17, p=0.001$, see Table 6 . As shown in Figure 11, the point clouds from the P4P had the smallest RMS error when registered to the FARO point cloud. 
Table 6

Descriptive Statistics of UAS Point Cloud Accuracy (RMS Error) by Aircraft

\begin{tabular}{lcccc}
\hline Aircraft & N & Mean & StDev & $95 \%$ CI \\
\hline P4P & 20 & 29.27 & 2.25 & $(26.61,31.92)$ \\
I2 & 20 & 30.92 & 3.54 & $(28.27,33.58)$ \\
I1 & 20 & 31.54 & 4.51 & $(28.89,34.20)$ \\
M1P & 20 & 33.41 & 5.36 & $(30.75,36.07)$ \\
Anafi & 20 & 34.47 & 3.36 & $(31.82,37.13)$ \\
BP2 & 20 & 36.29 & 6.03 & $(33.63,38.94)$ \\
M2ED & 20 & 36.44 & 11.75 & $(33.78,39.09)$ \\
\hline
\end{tabular}

Note. Measurements are in millimeters. P4P is the DJI Phantom 4 Professional; I2 is the DJI Inspire 2 equipped with an X5S and 15mm 1.7 ASPH lens; I1 is the DJI Inspire 1; M1P is the DJI Mavic Pro; Anafi is the Parrot Anafi; BP2 is the Parrot Bebop 2; M2ED is the DJI Mavic 2 Enterprise Dual.

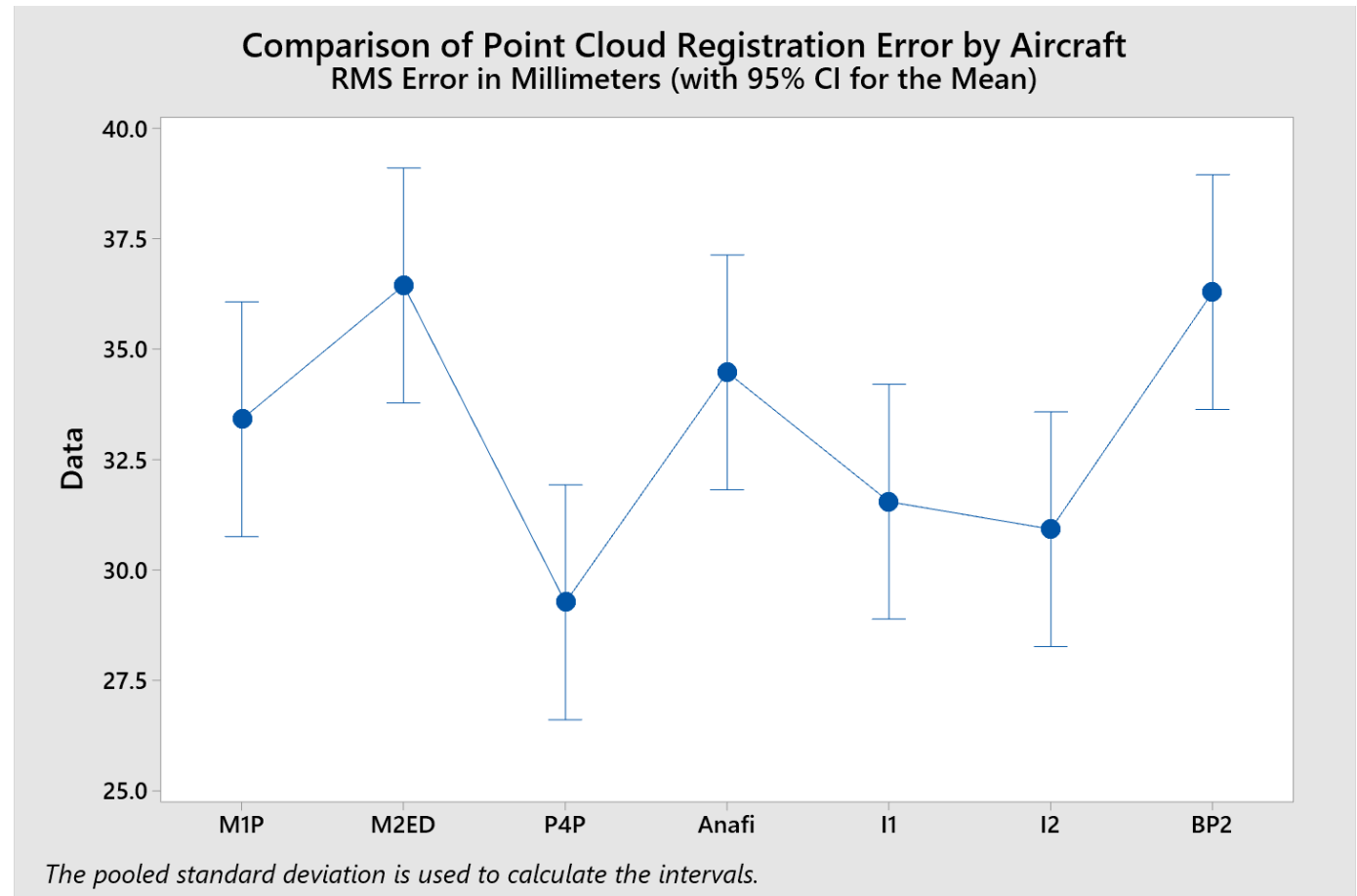

Figure 11. RMS error (in millimeters along the $\mathrm{Y}$ axis) of UAS point clouds by aircraft when registered to the FARO point cloud.

To determine which aircraft had the greatest precision to the FARO point cloud, a point distance information between UAS point clouds and the FARO point cloud was performed using the M3C2 plugin in CloudCompare. A comparison of 
point distances between the UAS point clouds and the FARO point cloud enabled a determination of which aircraft most closely compared (highest precision) to the FARO dataset. The subsampled core points from each UAS point cloud was grouped by aircraft. The $\mathrm{M} 3 \mathrm{C} 2$ plugin calculated the distance from each point in the UAS point to the closest point in the FARO point cloud and assigned a distance value.

A one-way ANOVA was conducted to compare the effect of the calculated point distances of UAS points by aircraft to the FARO points using the M3C2 plugin in CloudCompare. An analysis of variance showed an effect of UAS point clouds on FARO point cloud was significant, $F(6,2739221)=1030.45, p=0.000$, see Figure 12. The test results indicate there was a significant difference in the calculated distance by aircraft. The P4P had the smallest mean difference in calculated points between the UAS-generated point clouds and the FARO point cloud.

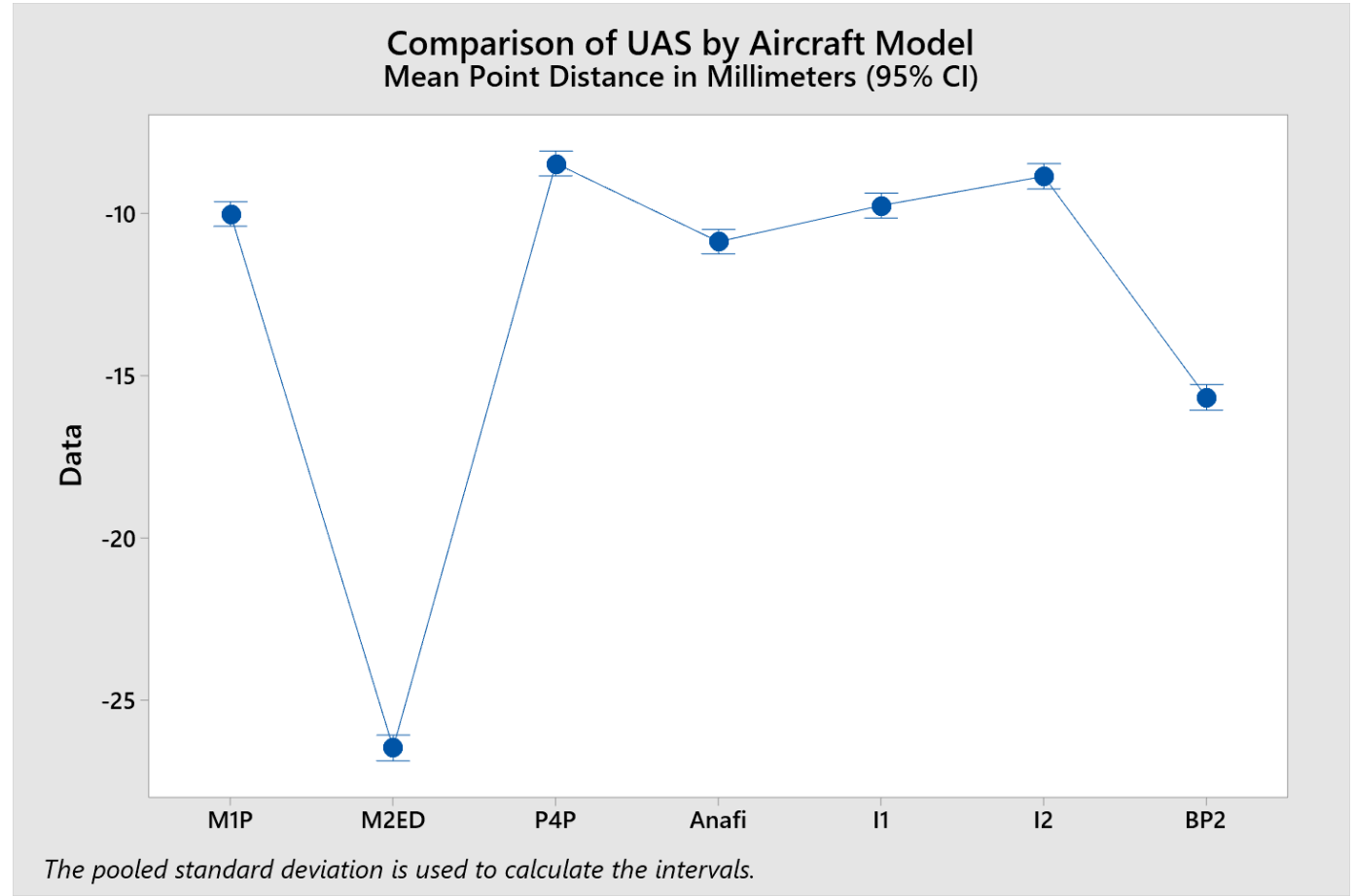

Figure 12. ANOVA test results showing the mean point distance calculation between UAS aircraft model to the FARO points. Differences are in millimeters. Points higher in the chart reflect a smaller calculated mean distance by aircraft model.

Conclusions and Recommendations

aUAS point clouds were not as accurate as the FARO scanner point cloud. The mean UAS point cloud RMS error of $33.2 \mathrm{~mm}$ from seven different UAS flying at five different flying heights and four different flight patterns and was 
significantly larger than the FARO point cloud accuracy of $2.6 \mathrm{~mm}$. The mean UAS point cloud density was 11,678 points per square meter, while the FARO point cloud had a density of 196,454 points per square meter. The mean UAS point spacing was $9.25 \mathrm{~mm}$, while FARO point spacing was $2.3 \mathrm{~mm}$. The UAS point clouds may still be accurate enough for forensic analysis at a crime scene or vehicle accident reconstruction.

There was a significant difference in flying height on the accuracy of the UAS point clouds. Flying at 82 feet, 100 feet or 150 feet resulted in smaller RMS errors than flying at 200 feet or 250 feet. Flying at 100 feet AGL yielded the highest precision of calculated point distances compared to the FARO point locations. Although these data revealed that as the flying height decreased, the RMS accuracy of the point cloud increased, but not significantly between 82 feet, 100 feet, or 150 feet. Additionally, flying at lower flying heights may not be practical over a crime scene because of obstacle clearance and other safety-related requirements. Based on these observations, a flying height of 100 feet AGL yielded the most precision and accuracy combined when compared to other flying heights.

There was a significant difference in UAS point cloud accuracy by the flight pattern when comparing UAS point clouds to the FARO point cloud. The double grid + circle flight pattern had smaller RMS errors compared to the other patterns. Using a multi-flight pattern, such as the double grid + circle, enabled Pix4Dmapper photogrammetry software to create more oblique-oriented points in the UAS point cloud. More oblique-oriented points in the UAS point cloud aligned to the predominantly oblique-oriented points in the FARO point cloud, because the FARO scanner was ranged between three and ten feet off the ground. Based on these observations, flying a UAS in the double grid + circle flight pattern had the most accuracy and precision when comparing the calculated point location between the UAS point clouds to the FARO point cloud.

There was a significant difference between UAS aircraft models when comparing RMS accuracy. The P4P had a smaller RMS error compared to other aircraft. The $\mathrm{P} 4 \mathrm{P}$ also had the highest precision with the smallest calculated point location between the UAS point clouds and FARO point cloud. The Inspire $2 \mathrm{had}$ nearly the same accuracy and precision as the $\mathrm{P} 4 \mathrm{P}$, indicating the potential of a higher resolution image sensor (20 megapixels for both the $\mathrm{P} 4 \mathrm{P}$ and $\mathrm{I} 2$ aircraft sensors) to contribute to 1) an increased density of points in a point cloud, 2) an increased RMS accuracy during the registration process, and 3) greater precision when comparing the calculated point distances between UAS and FARO point clouds. Based on these observations, using a 20-megapixel equipped sensor in a UAS, such as the P4P or I2, is recommended for crime scene reconstruction data collection from a UAS.

There was a substantial difference in the number of images captured, RMS accuracy and calculated point distance between the M2ED and other UAS equipped 
with rectilinear lenses (e.g., M1P, P4P, I1, I2, Anafi) at the same flying heights and similar flight patterns. Since the M2ED was controlled from the DJI Smart Controller control station, it was not possible to fly the M2ED using Pix4Dcapture as was the case for all other aircraft. Instead, the autonomous flight planning of the M2ED was performed using the DJI Pilot app. Further research is recommended to examine the differences of different flight planning software/ applications, such as Pix4Dcapture compared to DJI Pilot, and their contribution to RMS accuracy and calculated point differences.

It is also recommended for further research to examine the differences of RMS accuracy and calculated point locations between UAS point clouds and FARO point clouds with UAS equipped with LIDAR technology rather than the use of photogrammetry from visual images. Laser scanning technology equipped on a UAS can potentially provide faster data collection compared to a terrestrial laser scanner, such as the FARO scanner. There may also be a difference in density of point cloud points or point spacing from a LIDAR equipped UAS compared to an RGB sensor. 


\section{References}

Aeropoints (n.d.). Propeller Aeropoints: Make your drone data accurate. Retrieved from https://www.propelleraero.com/aeropoints/

Alidoost, F., \& Arefi, H. (2017). Comparison of uas-based photogrammetry software for $3 \mathrm{~d}$ point cloud generation: A survey over a historical site. ISPRS Annals of the Photogrammetry, Remote Sensing and Spatial Information Sciences, IV-4/W4, 55. doi:10.5194/isprs-annals-IV-4-W4-552017

Archuletta, M. (n.d.). "I am convinced the Grand Jury indicted because of FARO..." (Customer Testimony). Retrieved from https://insights.faro.com/ customer-quotes/i-am-convinced-the-grand-jury-indicted-because-of-faro

Araújo, P., Jr, Mendonça, M., Fontinele, J., \& Oliveira, L. (2019). Towards autonomous investigation of crime scene by using drones. Sensors \& Transducers, 234(6), 30-36. Retrieved from http://ezproxy.libproxy.db.erau.edu/login?url=https://search-proquestcom.ezproxy.libproxy.db.erau.edu/docview/2276750857? accountid=2720 3

Bullock, J. L., Hainje, R., Habib, A., Horton, D., \& Bullock, D. M. (2019). Public safety implementation of unmanned aerial systems for photogrammetric mapping of crash scenes. Transportation Research Record, 2673(7), 567574. https://doi.org/10.1177/0361198119850804

CloudCompare (n.d.). M3C2 plugin introduction. Retrieved form http://www.cloudcompare.org/doc/wiki/index.php?title=M3C2_(plugin)

Kovar, D. \& Bollo, J. (2018). Drone forensics. Digital Forensics Magazine. Retrieved from https://www.msab.com/download/case_studies/ Digital_Forensics_Feb-2018-MSAB.pdf

Dittrich, A., Weinmann, M., \& Hinz, S. (2017). Analytical and numerical investigations on the accuracy and robustness of geometric features extracted from 3D point cloud data. ISPRS Journal of Photogrammetry and Remote Sensing, 126, 195-208. doi:10.1016/j.isprsjprs.2017.02.012

DJI. (n.d.). Mavic 2 Enterprise dual specifications. Retrieved from https://www.dji.com/mavic-2-enterprise/specs

Elsner, P., Dornbusch, U., Thomas, I., Amos, D., Bovington, J., \& Horn, D. (2018). Coincident beach surveys using UAS, vehicle mounted and airborne laser scanner: Point cloud inter-comparison and effects of surface type heterogeneity on elevation accuracies. Remote Sensing of Environment, 208, 15-26. doi:10.1016/j.rse.2018.02.008

FARO. (n.d.). SCENE - The Most intuitive data scan software. Retrieved from https://www.faro.com/products/construction-bim/faro-scene/ 
Fiolka, T., Rouatbi, F., \& Bender, D. (2017). Automated detection and closing of holes in aerial point clouds using an uas. Gottingen: Copernicus $\mathrm{GmbH}$. doi:http://dx.doi.org.ezproxy.libproxy.db.erau.edu/10.5194/isprs-archivesXLII-2-W6-101-2017

Gabara, G., \& Sawicki, P. (2017). Study on 3d point clouds accuracy of elongated object reconstruction in close range - comparison of different software.

Vilnius: Vilnius Gediminas Technical University, Department of Construction Economics \& Property. doi:http://dx.doi.org.ezproxy.libproxy.db.erau.edu/10.3846/enviro.2017.18 8

Gabrlik, P., Cour-Harbo, A. 1., Kalvodova, P., Zalud, L., \& Janata, P. (2018). Calibration and accuracy assessment in a direct georeferencing system for UAS photogrammetry. International Journal of Remote Sensing, 39(1516), 4931-4959. doi:10.1080/01431161.2018.1434331

Galante, A., \& Cerreta, J. (2018, Spring). Integrating UAS into law enforcement operations. Tactical Edge, National Tactical Officer's Association.

Grenzdörffer, G. J., Niemeyer, F., \& Frank, A. (2015). symbiosis of uas photogrammetry and tls for surveying and $3 \mathrm{~d}$ modeling of cultural heritage monuments - a case study about the cathedral of St. Nicholas in the city of Greifswald. The International Archives of Photogrammetry, Remote Sensing and Spatial Information Sciences, XL(1), 91. doi:10.5194/isprsarchives-XL-1-W4-91-2015

Hosseinyalamdary, S. (2016). Traffic scene perception using multiple sensors for vehicular safety purposes (Doctoral Dissertation, The Ohio State University). Retrieved from https://etd.ohiolink.edu/pg_10?0::NO:10:P10_ ACCESSION_NUM:osu1462803166

IAFC. (2017). UAS regulatory/ops. International Association of Fire Chiefs. Retrieved from https://www.iafc.org/topics-and-tools/resources/resource /uas-regulatory-ops

Katz, E., \& Halámek, J. (2016). Forensic science: A multidisciplinary approach. Retrieved from https://ebookcentral.proquest.com

Kelvin, Lord William Thomson, Electrical Units of Measurement, lecture delivered to the Institution of Civil Engineers on May 3, 1883, in Popular Lectures and Addresses, Vol. 1, London: McMillan, 1889, p. 73.

Komar, D. A., Davy-Jow, S., \& Decker, S. J. (2012). The use of a 3-D laser scanner to document ephemeral evidence at crime scenes and postmortem examinations. Journal of Forensic Sciences, 57(1), 188-191. doi:10.1111/j.1556-4029.2011.01915

Lee, B. (2016). Protection, profit, or privacy: Exploring strategic solutions for integrating unmanned aerial systems (UAS) and the delicate balance between commercial opportunity and public safety. Homeland Security 
Affairs, Retrieved from http://ezproxy.libproxy.db.erau.edu/login? url=https://search-proquest-com.ezproxy.libproxy.db.erau.edu/docview/ 2206253996? accountid=27203

Liberadzki, P., Adamczyk, M., Witkowski, M., \& Sitnik, R. (2018). Structuredlight-based system for shape measurement of the human body in motion. Sensors (Basel, Switzerland), 18(9), 2827. doi:10.3390/s18092827

Lyu, N., Huang, G., Wu, C., Duan, Z., \& Li, P. (2017). Modeling vehicle collision angle in traffic crashes based on three-dimensional laser scanning data. Sensors, 17(3), 482. doi:http://dx.doi.org.ezproxy.libproxy.db.erau.edu/ $10.3390 / \mathrm{s} 17030482$

McFadden, C. (2018). Laser Scanning for Clues. Forensic Magazine. Retrieved from http://ezproxy.libproxy.db.erau.edu/login?url=https://searchproquest-com.ezproxy.libproxy.db.erau.edu/ docview/ 2052718346? accountid $=27203$

Mei, N. (2019). An approach to unmanned aircraft systems forensics framework (Order No. 13898531). Available from ProQuest Dissertations \& Theses Global. (2248709206). Retrieved from http://ezproxy.libproxy.db.erau.edu/login?url=https://search-proquestcom.ezproxy.libproxy.db.erau.edu/docview/2248709206? accountid=2720 3

Pix4D. (n.d.). Pix4Dcapture: Free drone flight planning mobile app. Retrieved from https://www.pix4d.com/product/pix4dcapture

Pix4D. (13 Aug, 2019). Drones, public safety \& emergency response: 5 use cases. Retrieved from https://www.pix4d.com/blog/emergency-response-dronemapping

Roder, A., Choo, K. R., \& Le-Khac, N. (2018). Unmanned aerial vehicle forensic investigation process: Dji phantom 3 drone as a case study. Retrieved mfrom https://arxiv.org/ftp/arxiv/papers/1804/1804.08649.pdf

Salamh, F. E., Karabiyik, U., \& Rogers, M. K. (2019). RPAS forensic validation analysis towards a technical investigation process: A case study of yuneec typhoon H. Sensors, 19(15).

doi:http://dx.doi.org.ezproxy.libproxy.db.erau.edu/10.3390/s19153246

Slocum, R. K., Parrish, C. E., (2017). Simulated imagery rendering workflow for UAS-based photogrammetric 3D reconstruction accuracy assessments. Remote Sensing, 9(4), 396. doi:10.3390/rs9040396

Struble, P. D., \& Donald E. (2013). Automotive accident reconstruction: Practices and principles (1st ed.). Baton Rouge, FL: CRC Press. doi: $10.1201 / \mathrm{b} 15542$

Thivierge, B. (2012). Vehicular accident reconstruction. In D. R. Franceschetti (Ed.), Applied Science (Vol. 5, pp. 1899-1905). Ipswich, MA: Salem 
Press. Retrieved from https://link-gale-com.ezproxy.libproxy.db.erau.edu/ apps/doc/CX2074500325/GVRL?u=embry\&sid=GVRL\&xid=93fbdb18

Torres-Sánchez, J., Torres-Sánchez, J., López-Granados, F., López-Granados, F., Borra-Serrano, I., Borra-Serrano, I., . . . Peña, J. M. (2018). Assessing UAV-collected image overlap influence on computation time and digital surface model accuracy in olive orchards. Precision Agriculture, 19(1), 115-133. doi:10.1007/s11119-017-9502-0

Turner, D., Lucieer, A., \& Wallace, L. (2014). Direct georeferencing of ultrahighresolution UAV imagery. IEEE Transactions on Geoscience and Remote Sensing, 52(5), 2738-2745. doi:10.1109/TGRS.2013.2265295

Urbanová, P., Jurda, M., Vojtíšek, T., \& Krajsa, J. (2017). Using drone-mounted cameras for on-site body documentation: 3D mapping and active survey. Forensic Science International (Online), 281, 52-62. doi:http://dx.doi.org.ezproxy.libproxy.db.erau.edu/10.1016/j.forsciint.2017 .10 .027 